\title{
Intricacies of Oral Malignant Melanoma - A Case Report
}

\author{
Misbah A. Tamboli ${ }^{1}$, R. S. Deshmukh ${ }^{2}$, P. U. Rao ${ }^{3}$, Nilima P. Narkhede ${ }^{4}$ \\ ${ }^{I}$ (Postgraduate student, Department of oral pathology, Bharati Vidyapeeth Dental college \& Hospital, India) \\ ${ }_{2}^{2}$ (Prof. \& Head, Department of oral pathology, Bharati Vidyapeeth Dental college \& Hospital, India) \\ ${ }^{3}$ (Reader, Department of oral pathology, Bharati Vidyapeeth Dental college \& Hospital, India) \\ ${ }^{4}$ (Postgraduate student, Department of oral pathology, Bharati Vidyapeeth Dental college \& Hospital, India)
}

\begin{abstract}
Malignant melanoma, also called as melanoma is one of the rare epithelial malignancies of oral cavity. The most common presentation of this neoplasia is ulceroproliferative growth along with pigmentations. Initially, these lesions are asymptomatic and painless, often demonstrating only discolorations. But they are aggressive, fast growing with a tendency to invade and metastasize. Here, this article presents one such case of oral malignant melanoma. Oral melanomas have poor prognosis and low survival rates and hence are important to know so as to provide early diagnosis and treatment.
\end{abstract}

Keywords: Gingiva, Hard palate, Oral melanoma, Oral malignant melanoma, Pigmentations.

\section{Introduction}

Malignant melanoma is one of the most unusual epithelial malignancies occurring in the oral cavity. It is the third most common malignant tumor of skin.(1) In oral cavity, it accounts for only $0.5 \%$ of all the malignancies and is regarded as extremely rare tumor.(2) Oral melanoma represent only $2 \%$ to $8 \%$ of all melanomas.(3) The primary malignant melanoma of oral cavity was first described by Weber in 1859.(4) These tumors mainly arise due to the uncoordinated and uncontrolled proliferation of the melanocytes that are present within the basal cell layer of the epithelium.(5) The most common sites affected are maxillary gingiva, hard palate, alveolar ridge.(6) These lesion clinically present as brownish-black macular or nodular growth and has to be differentiated from other pigmented lesions such as tattoos, melanotic macules, melanocytic nevus, drug induced pigmentation, some of the pigmented vascular lesions, and oral pigmented lesions associated with endocrine disorders or different syndromes.(7)(8) Hence histopathological examination is mandatory for diagnosis. This article intends to describe a case and the characteristics of malignant melanoma of oral cavity.

\section{Case Report}

A sixty three year old female patient complained of pain and swelling in the upper front region of jaw since a month. She presented with progressive ulceroproliferative enlargement on attached and marginal gingiva of maxillary anterior region extending from 14 to 24 region. Lobulated, extrabony, erythematous, soft tissue growth with brownish black pigmentations was noticed.(Fig. 1) On palpation, the lesion was soft, friable and the associated teeth were mobile. Bleeding was observed on touch. Blackish discolorations of mucosa were also observed over the palate, right buccal mucosa and labial mucosa.(Fig. 2) Patient also gave history of hypertension since 15 years and mishri application for 40 years. She was under medication (calcium channel blockers) for hypertension. Provisional diagnosis of malignant melanoma, melanoacanthoma and oral melanosis associated with drug induced gingival enlargement were given and incisional biopsy was taken for the lesional tissue to confirm the diagnosis.

Histopathological examination revealed epithelium and connective tissue. Epithelium was stratified squamous parakeratinized in nature. The epithelium showed proliferation of large ovoid (epitheloid) and spindle shaped pleomorphic cells with perinuclear vacuolization.(Fig. 3) These cells were arranged in whorling pattern typical of pagetoid appearance. The epithelial cells also showed dysplastic features such as nuclear and cellular pleomorphism, increased nuclear cytoplasmic ratio, increased and abnormal mitotic activity. These atypical cells presented with brownish pigmentation suggestive of melanin.(Fig. 4) The atypical epitheloid and spindle shaped cells invaded the connective tissue in the form of strands, cords and sheets and fascicular pattern demonstrating vertical growth pattern.(Fig. 5) The connective tissue showed coalescing of the collagen fibres, dilated blood vessels and chronic inflammatory cell infiltrate chiefly plasma cells and lymphocytes. Abundant areas of necrosis were evident. As the histopathological picture was suggestive of malignant melanoma, Immunohistochemical staining was done for melanocyte specific marker HMB-45. Immunohistochemical analysis showed the tumor cells positive for marker HMB-45.(Fig. 6) Hence the final diagnosis of malignant melanoma was given. 


\section{Discussion}

Introduction: Oral melanoma is a rare malignant disease, which represent only $2 \%$ to $8 \%$ of all melanomas.(3) Melanoma was first described by Weber in 1859 and later was named as "melanotic sarcoma" by Lucke in 1869.(9) About more than $20 \%$ of all melanomas occur in the head and neck region.(10) It is relatively more frequent in countries like Japan, Uganda, and India.(11) The mean age of occurrence is approximately about 60 years, with a wide range of about 20-90 years.(12) Melanoma is reported to show slight male predominance but some studies have been reported higher gender distribution of mucosal melanoma for women while others indicate that there is no significant difference between sexes in the incidence of this tumor.(13) The most common sites for oral melanoma are hard palate and maxillary gingiva.(14)

Etiopathogenesis: This tumor is of melanocytic origin. Melanocytes are the neural crest-derived cells that are present within the basal cell layer of the skin and mucous membrane.(15) The oral melanoma is thought to arise either de novo from melanocytes or from the pre-existing oral pigmentations.(16) As in the present case also, patient reportedly had nevi in the palatal region which may have transformed into the melanoma. The etiologic factors such as exposure to sunlight, denture irritation, chewing tobacco with betel nut, and smoking have been considered.(17) The risk factors in developing malignant melanoma includes pale skin, blond or red hair, numerous freckles and tendency to tan, presence of more than 50 acquired nevi, more than five atypical nevi, large congenital nevi, nevi larger than 6mm, PUVA therapy, use of tanning salons, Xeroderma pigmentosum, Immunosuppression, chemical exposures, scars, Marjolin's ulcer and genetic factors.(18) The cytogenetic alteration acquired within the oncogenes, tumor-suppressor genes and DNA repair genes of the melanocytes are said to play a role in the etiopathogenesis of melanoma.(13) Transformation of normal melanocytes into malignant cells is still unclear but Up- or down- regulation of various effectors involved in different molecular pathways appears to be the cause for this transformation.(18) The inadequate regulation of biosynthesis of melanin by the melanocytes leads to increased oxidative stress and metabolic by-products that cause DNA damage in the affected melanocytes and thus favor cancer initiation and progression.(13) Molecular studies in melanoma have demonstrated BRAF mutations along with involvement of p53 and p16 tumor suppressor genes.(18)(19) S100A1, S100B, Bcl-2, and CD44 are said to have a role in transformation of melanocytes to melanoma cells and Genes, such as MC1R (Melanocortin1 Receptor) and DNA repair genes, are found to be important in determining susceptibility for melanoma in the general population.(18)

Clinical features: Oral melanomas may present as painless, dark brown or black colored, sometimes erythematous macular or nodular growth with or without ulceration.(7) The melanomas show clinical features that can be described with characteristic $\mathrm{ABCDE}$ rule of melanomas where A stands for asymmetry, in which one half of the lesion does not match with the other, B for borders that show irregular blurred, notched or ragged edges, $\mathrm{C}$ is for color, usually non-uniform brown, black, tan, red, white or blue pigmentations, D stands for diameter of lesion, that is usually greater than $6 \mathrm{~mm}$ and $\mathrm{E}$ is for elevation or a raised surface.(17) Non pigmented lesions of melanoma called Amelanotic melanoma have also been reported.(20) Based on the clinical appearance, oral melanomas are classified by Padhye and D'Souza into five types: pigmented nodular, non pigmented nodular, pigmented macular, pigmented mixed, and non pigmented mixed.(17) The case described here can be considered as pigmented mixed type. The patient may present with symptoms like swelling, bleeding, pain, tooth mobility, ill-fitting dentures and delayed healing of post extraction wounds.(21) However, initially melanomas are asymptomatic and often go unnoticed by the patient contributing delay in diagnosis.(15) Because of its biologically aggressive nature and delayed diagnosis, the prognosis of these lesions is poor.(22) The 5-year survival rates range from $6.6 \%$ to $20.0 \%$.(7)

Histopathological examination: reveals the connective tissue stroma is infiltrated by islands of proliferating spindle, plasmacytoid and epitheloid shaped tumor cells showing nuclear hyperchromatism, pleomorphism, altered nuclear cytoplasm ratio and abundant brownish pigmentations suggestive of melanin.(23)(24) The malignant cells are typically arranged in solid, alveolar, pagetoid or organoid pattern.(25) Malignant melanomas can show both vertical and radial growth patterns. In radial growth pattern, the melanocytes have a propensity to spread horizontally through the basal cell layer of epithelium while vertical growth pattern shows vertical invasion of malignant melanocytes within the connective tissue.(26) Radial growth phase is usually microinvasive, wherein few malignant cells are seen in superficial papillary dermis and vertical growth phase usually shows cells that have entered the tumorigenic and/or mitogenic phase.(18) Mucosal melanomas usually present in a vertical growth (nodular) phase of disease and are more aggressive in nature.(4) In oral melanomas, vascular invasion is seen more commonly than perineural invasion but metastatic melanomas can show both vascular and perineural invasion.(25) The case reported in this article also showed atypical epitheloid and spindle shaped cells along with pagetoid appearance and vertical growth phase pattern. The oral mucosal melanomas can be classified histopathologically as in situ, invasive, and combined (both in situ and invasive).(4) The in situ pattern is one in which the neoplastic growth is limited to the epithelium and the epithelial-connective tissue interface while an invasive pattern is where the neoplasm is seen within the underlying connective tissue.(27) The in situ melanomas show proliferation of atypical melanocytes with 
angular, hyperchromatic nuclei.(28) Invasive pattern show a variety of cell types such as spindle, epithelioid, plasmacytoid, and clear cells with large, vesicular nucleus and prominent nucleoli.(28) The atypical cells in invasive pattern are usually arranged into sheets or organoid/alveolar formation, while in the in situ pattern, the cells are arranged irregularly at the epithelial connective tissue interface or may be distributed in aggregates.(28) Most of the oral melanomas (about 55\%) demonstrate the combined pattern of growth, but the in situ pattern and the invasive pattern is also seen in about $15 \%$ and $30 \%$ of the oral melanomas respectively. (28)

Immunohistochemistry: As melanoma show wide spectrum of histological features that mimic epithelial, mesenchymal and neural tumors, immunohistochemical analysis becomes necessary for diagnosis.(25) Various immunohistochemical markers used for melanomas are NKI/C-3, S- 100 protein, gp100 (HMB-45), Mart-1 (Melan-A), vimentin, tyrosinase and microphthalmia transcription factor (MiTF) but HMB45 is a more specific marker for melanoma.(24) The confirmatory diagnosis for the present case was done using HMB-45 marker.

Treatment: Wide surgical resection with margin of 2 to $5 \mathrm{~cm}$ is the most effective treatment for melanoma, but it is difficult to achieve for oral melanomas because of anatomical reasons.(8)

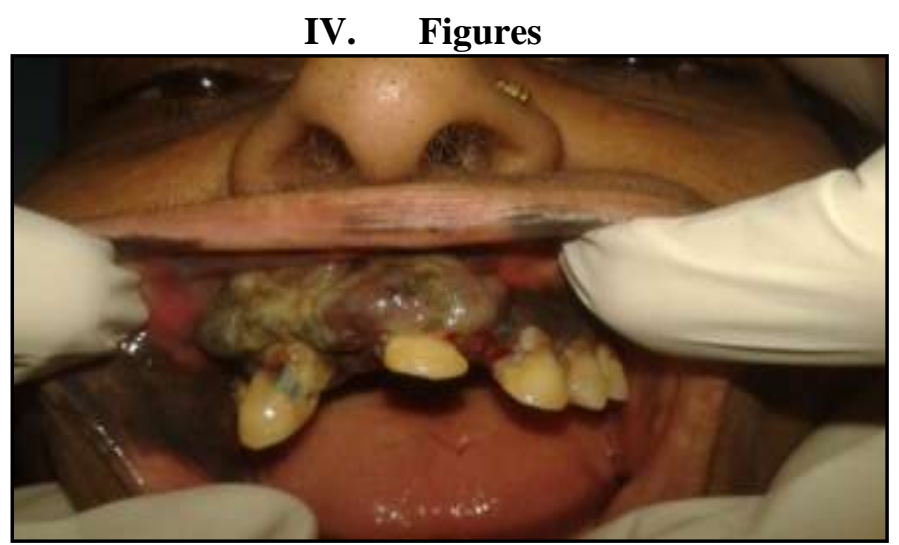

Figure 1. Pigmented gingival swelling

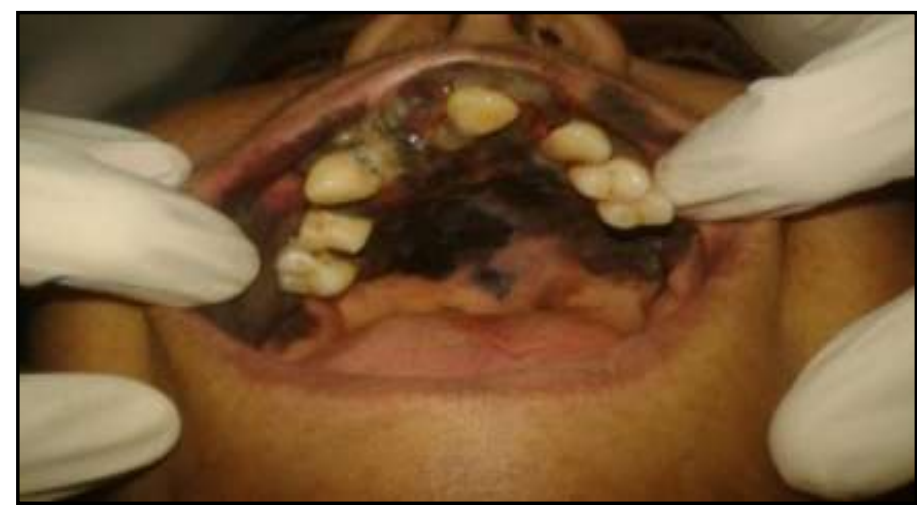

Figure 2. Pigmentations on palate and buccal mucosa

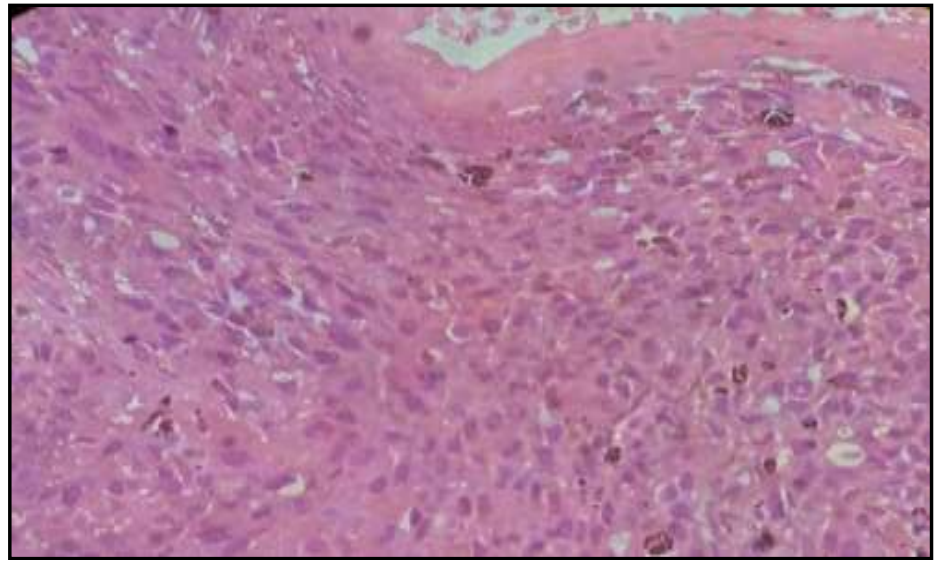

Figure 3. Proliferating epitheloid and spindle shaped 


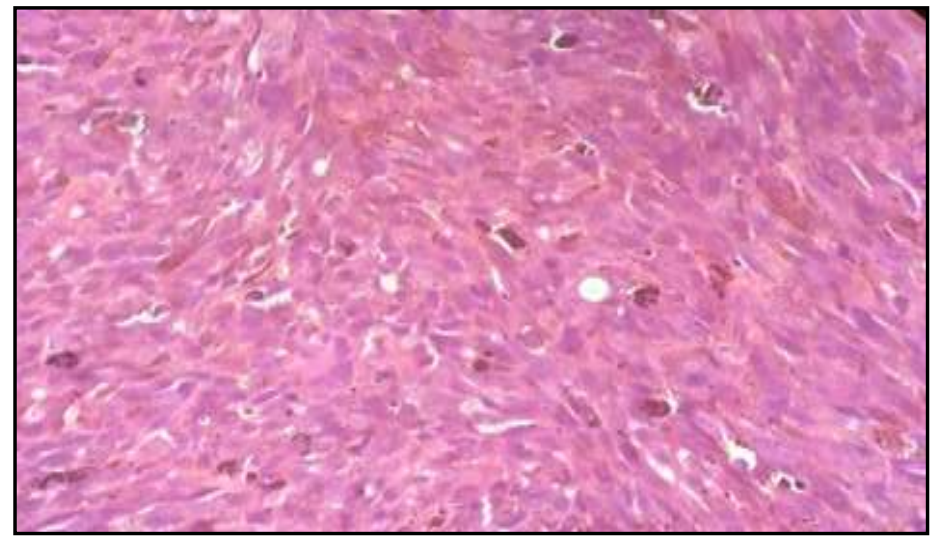

Figure 4. Atypical cells with melanin pigmentations

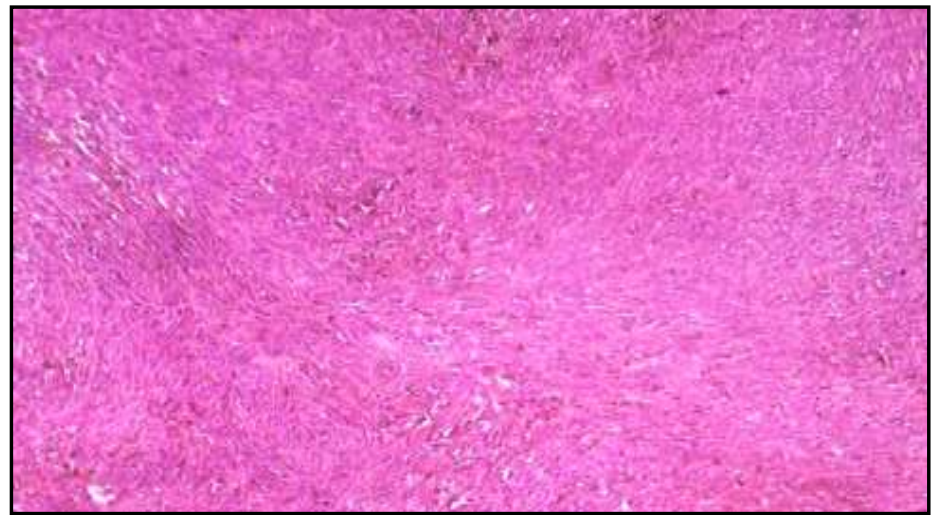

Figure 5. Tumors cells arranged in fascicular pattern

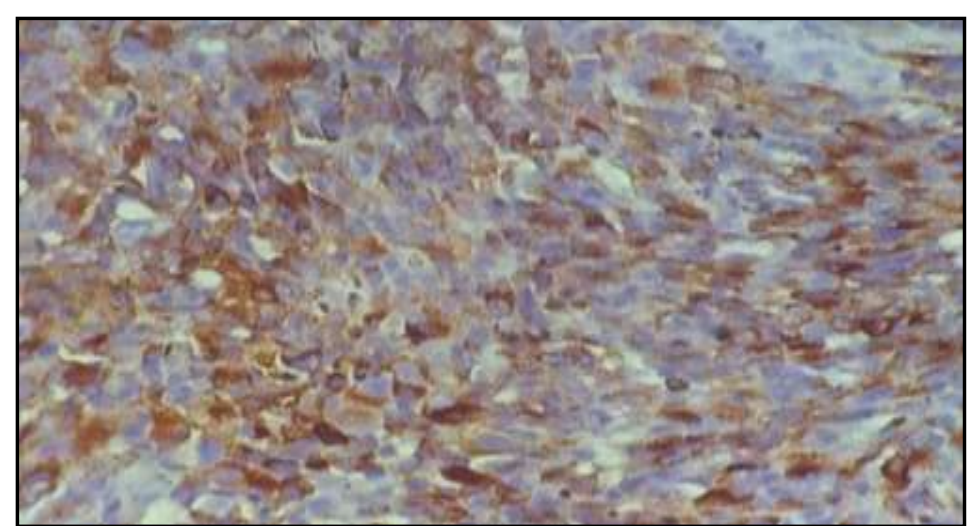

Figure 6. IHC demonstrating tumor cells positive for HMB-45.

\section{Conclusion}

Oral mucosa often presents with a variety of pigmented lesions, from which the malignant and the most aggressive ones such as melanomas needs to be differentiated. Biopsy should be mandatory for all the lesions that clinically comply the $\mathrm{ABCDE}$ rule of malignant melanomas so as to confirm the lesion histopathologically and provide the early necessary treatment.

\section{References}

[1]. Uratani AM, Perez DE da C, Vargas PA, Jorge J. Oral melanoma : review of the literature. Braz J Oral Sci. 2004;3(9):428-32.

[2]. Belhoucha B, Essaadi Z, Benhommad O, Rochdi Y, Nouri H. Primary Malignant Melanoma of the Oral Mucosa : Report of an Unusual Case. Int J Dent Sci Res. 2014;2(4):103-5.

[3]. Elomrani F, Mouzount H, Ouziane I, Khmamouch R, Boukir A, Elkabous M. Melanoma of the Oral Cavity : About Two Cases and Review of Literature. Int J Clin Med. 2013;2013(April):191-4.

[4]. Biradar V, Latturiya R, Biradar S. Late Diagnosis of Oral Mucosal Melanoma : Case Report. J Dent Allied Sci. 2012;1(2):85-7.

[5]. Dimitrakopoulos I, Lazaridis N, Skordalaki A. Primary malignant melanoma of the oral cavity . Report of an unusual case. Aust Dent J. 1998;43(6):379-81. 
[6]. Anupama I V, Chandran D, Girija KL, C TB, Balan A. Intraoral Malignant Melanoma - Concise Appraisal Of A Precarious Neoplasm. IOSR J Dent Med Sci. 2016;15(2):24-8.

[7]. Khalifa H, Abdullah S, Sallam K, Khalil H, Moneim IA, Elaffandi A. Primary malignant melanoma of the tongue. Can J Surg. 2009;52(6):309-11.

[8]. Chiu T, Lin H, Su C, Huang C. Primary Malignant Melanoma of the Tongue. Chang Gung Med J. 2002;25(11):764-8.

[9]. Elneel Ahmed MA, Karrar MA, El-Siddig AA, Zulfu A. Case report Oral malignant melanoma : a rare case with unusual clinical presentation. Pan Afr Med J. 2015;8688:1-5.

[10]. Ahmadi-motamayel F, Falsafi P. Report of a rare and aggressive case of oral malignant melanoma. Oral Maxillofac Surg. 2013;17:47-51.

[11]. Hashemi Pour M. Malignant melanoma of the oral cavity: A review of literature MS. Indian J Dent Res. 2008;19(1):47-51.

[12]. Nascimento J de S do, Taylor AM, Almeida OP de, Andrade BAB de. Clinical \& Experimental Pathology Primary Oral Melanoma : A Case Report with Immunohistochemical Findings. J Clin Exp Pathol. 2014;4(5):6-8.

[13]. Hendzel OW, Słowińska M, Olszewska M, Rudnicka L. Melanoma of the oral cavity : pathogenesis , dermoscopy , clinical features, staging and management Pathogenesis Epidemiology. J Dermatol Case Rep. 2014;3:60-6.

[14]. Lamichhane NS, An J, Liu Q, Zhang W. Primary malignant mucosal melanoma of the upper lip : a case report and review of the literature. BMC Res Notes [Internet]. BioMed Central; 2015;1-10. Available from: "http://dx.doi.org/10.1186/s13104-015-1459-3

[15]. Twyana R, Hong Z. Malignant Melanoma In The Oral Cavity : A Rare Case Report With Review of Literature. J Nepal Dent Assoc JNDA. 2015;15(1):52-8.

[16]. Pai A, Prasad S, Patil BA, Dyasanoor S, Hegde S. Oral pigmentation: Case report and review of malignant melanoma with flow charts for diagnosis and treatment. Gen Dent. 2012;410-6.

[17]. Rani GS, Kumar TV, Kolasani B, Begum R, Srinivasan AP. Case Report Primary Malignant Melanoma of Maxilla : Report of a Case with Discussion. Case Rep Dent. 2014;2014.

[18]. Bandarchi B, Ma L, Navab R, Seth A, Rasty G. From Melanocyte to Metastatic Malignant Melanoma. Dematology Res Pract. 2010;2010.

[19]. Uong A, Zon LI. Melanocytes in Development and Cancer. j cell physiol. 2010;222(1):38-41

[20]. Gottipati P, Sangeetha S, Channabasappa B, Deepak V, David MP. Case Report Oral Mucosal Amelanotic Melanoma : A Rare Case Report. IJSS Case Reports Rev. 2014;1(6):6-9.

[21]. Hsiao K, Liu C, Wong T, Chen K. Oral Mucosa Malignant Melanoma : Clinical Features , Diagnosis , Treatment , and a Case Report. J Dent Probl Solut. 2015;2(2):19-24.

[22]. Vinuta H, Kaveri H. International Journal of Medical Research \& Health Sciences. Int J Med Res Heal Sci. 2014;3(3):726-30.

[23]. Reddy J, Kanth R, Kumar S, Swathi R, Madhavi A. Oral Malignant Melanoma. J Clin Diagnostic Res. 2015;9(1):11699.

[24]. Kaur J, Sood K. Malignant Melanoma of the Oral Cavity : A Review. Int J Sci Res. 2015;4(9):2013-5.

[25]. León J, Contreras E. Primary oral melanoma : A histopathological and immunohistochemical study of 22 cases of Latin America. Med Oral Patol Oral Cir Bucal. 2012;17(3):15-20.

[26]. Neville B, Damm DD, Allen CM, Bouquot JE. Oral and Maxillofacial Pathology. Elsevier; 2009. 433-437 p.

[27]. Padhye A, Jovita D. Oral malignant melanoma : A silent killer ?? J Indian Soc Periodontol. 2011;15(4):1-4.

[28]. Mücke T, Borgmann A, Wolff K, Mitchell DA. Oral Malignant Melanoma. In: Morton MR, editor. Treatment of Metastatic Melanoma [Internet]. InTech; 2011. Available from: http://www.intechopen.com/books/treatment-of-metastatic-melanoma/oralmalignant-melanoma 